\title{
On the Zero-Level of the Soft X-ray Background
}

\author{
M.J. Freyberg \\ Max-Planck-Institut für extraterrestrische Physik, D-85740 Garching, Germany
}

\begin{abstract}
The soft X-ray background (SXRB, $0.1-2.0 \mathrm{keV}$ ) is generally believed to be comprised of a local component, the Local (Hot) Bubble (LHB), which is only marginally absorbed, and of distant emission (galactic halo, extragalactic background). It is vital to know the foreground emission (spectrum, intensity) if one wants to disentangle and determine the 3-dimensional structure of the X-ray emitting regions. During the ROSAT PSPC All-Sky Survey non-cosmic enhancements of the measured X-ray count rate have been observed. These lasted typically for up to 8 hours (i.e. a couple of orbits). Using PSPC data it has been shown that these so-called "long-term enhancements" (LXE) are related to geomagnetic storms and variations of the solar wind. Moreover, during an observation of the comet Hyakutake indications for a correlation of the X-ray background with the X-ray brightness of the comet have been found. This can be interpreted in terms of an X-ray emitting region around the Earth. Although the variable component of these enhancements could be subtracted from the sky maps an uncertainty remained concerning an undetected and relatively constant contribution which might be present in soft X-ray observations. As a consequence this could explain or contribute to the high PSPC count rate observed from the dark side of the Moon.
\end{abstract}

\section{Introduction}

During the ROSAT PSPC All-Sky Survey non-cosmic enhancements of the measured X-ray count rate have been observed. They lasted typically for up to 8 hours (i.e. a couple of orbits) and were called "long-term X-ray enhancements" (LXE). As the sky was scanned in great circles ( 1 orbit $=1$ circle $\sim 96 \mathrm{~min})$ the enhancements affect the whole strips of the sky $\left(\sim 360^{\circ} \times 2^{\circ}\right)$ during the period. According to Snowden et al (1992) the LXE are a "nonlocal effect". Variable and/or high background values in previous survey missions (e.g., Wisconsin, HEAO 1 A2, EUVE) are also noted by, e.g., McCammon et al (1983), Garmire et al (1992), Jelinsky, Vallerga \& Edelstein (1995). Using PSPC data from the Survey as well as from the Pointing Programme it has been shown (Freyberg 1994) that the LXE are related to geomagnetic storms and variations of the solar wind. Events from an LXE appear to be vignetted, i.e. have passed the X-ray optics. The X-ray spectrum is soft and hardens if the LXE is stronger. Within the PSPC resolution acceptable fits can be obtained with a thermal spectrum (Raymond-Smith plasma with solar abundances $)$, however, significant foreground absorption $\left(\sim 10^{19} \mathrm{~cm}^{2}\right)$ were required, which left some doubt on the physical relevance of the fit. There are significant variations in intensity and spectrum within an individual orbit. 
LXEs are also present at low geomagnetic latitudes. Accompanied is an additional component at high geographic (geomagnetic) latitudes which seems to be related to geomagnetic substorms. Although the variable component of these enhancements could be subtracted from the sky maps (Snowden et al 1995) an uncertainty remained concerning an undetected and relatively constant contribution which might be present in all ROSAT X-ray observations.

\section{Does the Earth Act as an X-ray Bright Comet?}

In 1996 a new field in X-ray astronomy was born with the detection of soft $\mathrm{X}$-rays from Comet C/Hyakutake 1996 B2 (Lisse et al 1996). The emission was found to be unexpectedly bright in X-rays and offset from the comet nucleus. In a recent model, Cravens (1996) suggested that the X-rays are generated by charge transfer collisions of high charge state solar wind heavy ions with cometary neutrals. Almost the full field-of-view of the ROSAT HRI was filled with the comet image. Therefore the total extent cannot be accurately determined. A ROSAT WFC image indicates a size of more than $1^{\circ}$ (Lisse et al 1996). During the 9 observation intervals variations of the $\mathrm{X}$ ray count rate have been observed ("outburst" by a factor of $2-5$ ). However, further detections of comets in the RASS have shown that besides outbursts a general (zero-level) emission is present. The question is whether the Earth is surrounded by such an X-ray emitting region as well. One way to answer this is to look for background intensity variations that seem to be correlated with the X-rays from the comet. During the comet observations in March 1996 a calibration of the HRI background was performed. Observations with typical cosmic X-ray background values $\left(3-4 \times 10^{3} \mathrm{cts} / \mathrm{Ms} / \operatorname{arcmin}^{2}\right)$ have been selected (e.g. omitting the LMC). Periods of excessively high background occuring at high geographic latitudes of the orbit were excluded; point-like as well as extended sources had been cut out by standard procedures. Finally, only observation intervals were chosen with more than 600 seconds to have sufficient signal-to-noise ratio.

\section{$3 \quad$ HRI Background Excess and Zero-Level}

In Fig.1 we present the ROSAT HRI X-ray light-curves of Hyakutake (Lisse et al 1996) as well as of the X-ray background (for more details see Freyberg, Dennerl \& Trümper 1997). The times of comet observations are indicated by dashed lines. The data sets of the background indicate an increase of the count rate close to the outburst. This suggests that variations in the solar wind not only affect the comet's X-ray emission but also the one of the Earth. This excess is of the order of $\sim 500 \mathrm{cts} / \mathrm{Ms} / \mathrm{arcmin}^{2}$ (i.e. $\sim 15 \%$ ). Assuming that this variation can be scaled to the comet's outburst variation (i.e. if the outburst is mainly due to solar wind variation and not a cometary 

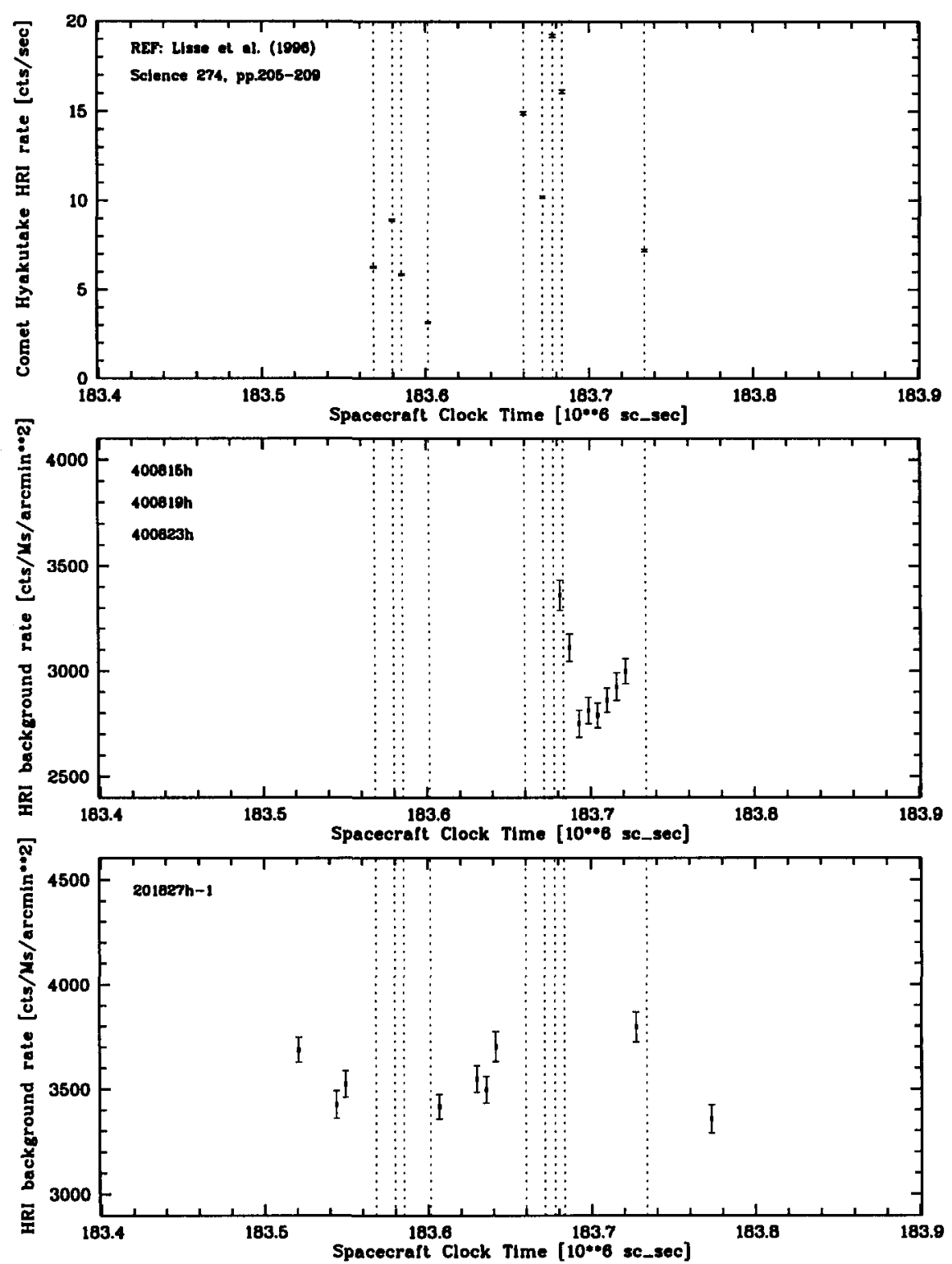

Fig. 1. Top: HRI count rate of Comet C/Hyakutake 1996 B2 according to Tab.1 of Lisse et al (1996). Dashed lines indicate the times of the individual observations of the comet. An outburst is visible in the $5^{\text {th }}-8^{\text {th }}$ observation. Middle: HRI background count rate of 3 observations towards $(l, b) \sim(30,0)$, with exclusion of sources, excessively high background at high geographic latitudes, short intervals. Variations of several $10^{-4}$ counts/s/arcmin ${ }^{2}$ are visible. Bottom: HRI background count rate of one observation towards $(l, b) \sim(30,17)$, similar to above. 
effect) then a zero-level enhancement of $\sim 10^{2} \mathrm{cts} / \mathrm{Ms} / \mathrm{arcmin}^{2}$ for the HRI background can be derived. According to Lisse et al (1996) the cometary spectrum is consistent with $k T \sim 0.2-0.7 \mathrm{keV}$. The actual spectrum probably consists of a large number of lines (Cravens 1997) and cannot be distinguished from continuum emission by ROSAT. For a Raymond-Smith-type plasma the HRI zero-level excess can be translated into a PSPC zero-level excess of $\sim 3(1.5-5)$ times the HRI value.

\section{Consequences and Conclusions}

What are consequences of an additional background $\sim 300 \mathrm{cts} / \mathrm{Ms} / \mathrm{arcmin}^{2}$ for PSPC observations? The origin of this component is very local $\left(\sim 10^{5} \mathrm{~km}\right)$ and thus be in front of any cosmic source, e.g. any X-ray shadow. One famous - shadow has been cast by the Moon's dark side onto the X-ray background (Schmitt et al 1991). It was found that the dark side of the Moon was brighter than expected by a factor of more than 10 , namely of the order of $\sim 300 \mathrm{cts} / \mathrm{Ms} / \mathrm{arcmin}^{2}$. This excess is consistent with the effect of an X-ray emitting region around the Earth. The dark lunar spectrum is consistent with an cometary spectrum of $k T \sim 0.2 \mathrm{keV}$ (Freyberg, Schmitt \& Snowden 1997; Freyberg, Dennerl \& Trümper 1997). Finally, we want to note, that the results of the present analysis are preliminary and provide only an order-of-magnitude estimate. One purpose was to propose a possible extra component to the SXRB.

\section{References}

Cravens T.E.: 1996, Geophys.Res.Lett. 24, 105

Freyberg M.J.: 1994, PhD thesis, LMU München

Freyberg M.J., Schmitt J.H.M.M., Snowden S.L.: 1997, in preparation

Freyberg M.J., Dennerl K., Trümper J.: 1997, in preparation

Garmire G.P., Nousek J.A., Apparao K.M.V., Burrows D.N., Fink R.L., Kraft R.P.: 1992, ApJ 399, 694

Jelinsky P., Vallerga J., Edelstein J.: 1995, ApJ 442, 653

Lisse C.M., Dennerl K., Englhauser J., Harden M., Marshall F.E., Mumma M.J., Petre R., Pye J.P., Ricketts M.J., Schmitt J.H.M.M., Trümper J., West R.G.: 1996, Science 274, 205

McCammon D., Burrows D.N., Sanders W.T., Kraushaar W.L., 1983, ApJ 269, 107

Schmitt J.H.M.M., Snowden, S.L., Aschenbach B., Hasinger G., Pfeffermann E., Predehl P., Trümper J.: 1991, Nature 349, 583

Snowden S.L., Plucinsky P.P., Briel U. Hasinger G., Pfeffermann E.: 1992, ApJ 393, 819

Snowden S.L., Freyberg M.J., Plucinsky P.P., Schmitt J.H.M.M., Trümper J., Voges W., Edgar R.J., McCammon D., Sanders W.T.: 1995, ApJ 454, 643 